\title{
COMBINING ABILITY AND GENETIC COMPONENT ANALYSES FOR WHEAT UNDER WATER STRESS AND NORMAL IRRIGATION
}

\author{
E.H. El-Seidy ${ }^{(1)}$, A.A. El-Gammaal ${ }^{(1)}$, R.M. Koumber ${ }^{(2)}$ \\ and Hend M. Abdallah ${ }^{(1)}$ \\ ${ }^{(1)}$ Faculty of Agriculture, Agronomy Department,Tanta University, Egypt. \\ (2) Agriculture Research Centre.
}

Received: Sep. 1, 2018

Accepted: Sep. 25, 2018

ABSTRACT: The aim of this study was to assess the variations among set a half diallel cross using eight varieties for drought characters, estimating combining ability and genetic components. For this objective, the investigation was carried out at the Experimental Farm of Etay El-baroud Agricultural Research Station during the two seasons of 2015/2016and 2016/2017, Eight diverse wheat varieties (Triticum aestivum, L.) and $28 F_{1}$ 's were planted in two experiments. The first experiment was normally irrigated 4 irrigations (Non stress) and the second one was irrigated one time after planting irrigation (Stress). General (GCA) and specific (SCA) combining ability mean squares were highly significant for all studied traits in both environments as well as the combined analysis. Such results indicated that both types of combining ability are important in the inheritance of these traits.It could be concluded that the parent P7 (Giza 171) seemed to be the best general combiner for grain yield/ plant and most of yield components.

$N$. kernel / spike in both exuberant and the combined only s, 1000 kernel weigh and harvest index in the drought treatment and the combined ability and grain yield in the normal irrigation.

The best sij effects were detected for the crosses P6 (Misr1 )x P7 (Giza171), P7 x P8 (Giza168) and P1 (Gemmeiza) x P6 Misr 1 at both conditions and their combined for grain yield and some traits stadied.

Key words: Triticum aestivum, Drought, GCA, SCA, Stress, Water, Tolerance, wheat

\section{INTRODUCTION}

Wheat (Triticum aestivum, L.) is the major cereal crop in Egypt as well as several other countries. World average cultivated area of wheat reached 221.73 million hectares in 2017; the total production was $751.36^{*}$ million metric tons, with an average productivity of 3.39* metric tons per hectare. Egypt grew in 2017, 1.25* million hectares that produced 8.10* million metric tons of grains, with an average yield of $6.43^{*}$ metric tons per hectare. With increasing population, it could hardly satisfy only $55 \%$ of local requirements. The increasing gap between production and consumption necessitates increasing wheat production in Egypt. To overcome this problem is to increasing the productivity of wheat through an efficient breading program.

Drought-resistant genotypes are able to maintain metabolic activities in their tissues with low water potential (Sairam et al., 1990). Drought resistance in genotypes recently developed through breeding programs is mostly related to the plant's ability to protect itself from water loss under dry conditions, rather than plant tolerance against water loss. Protection from water loss is a result from different structural characteristics 
(root length, seedling power, plant height, leaf area, flowering duration, etc.) related to plant development phenology and physiology (Blum, 2006). In environments where drought is experienced during the early growth periods, plant characteristics able to ensure germination-emergence and survival of seedlings should be taken into consideration (Monneveux and Ribaut, 2006).

Plant breeders focus on development of high yielding wheat cultivars by crossing good general combining lines and selecting desirable transgressive segregants from resulting hybrids for grain yield and other traits. Some researchers determined that the general combining ability effects for yield and other characters have played a significant role in selecting parents for grain yield (Akbar et al., 2009).

The knowledge of combining ability is useful to assess differences among the genotypes and also, elucidate the nature and magnitude of gene actions involved. It has an important role to select parents and crosses and it helps to decide breeding methods to be followed to choose desirable individuals (Salgotra et al., 2009).

The major objectives of the present investigation therefore to estimate gene action and the importance which should be given to these materials in a breeding program.

\section{MATERIALS AND METHODS}

The investigation was carried out at the Experimental Farm of Etay El-baroud Agricultural Research Station during the two seasons of 2015/2016and 2016/2017, Eight diverse wheat varieties (Triticum aestivum, L.) and $28 F_{1}$ 's were planted in two experiments. Table (1).
In 2015/16 growing season, grains from each of the parental varieties were sown at a various sowing dates in order to overcome the differences in time of heading. During this season, all possible parental combinations without reciprocals were made among eight parents giving a total of twenty-eight crosses. In 2016/2017season, the eight parents and their twenty-eight possible $F_{1}$ crosses were sown on $17^{\text {th }}$ Nov. 2016. Two adjacent experiments were conducted. The first experiment was normally irrigated 4 irrigations (Non stress) and the second one was irrigated one time after planting irrigation (S tress). Each experiment was designed in a randomized complete block design with three replications. Each plot consisted of one ridge; three meters long with $20 \mathrm{~cm}$ between ridges and plants within ridge were $20 \mathrm{~cm}$. apart allowing a total of 15 plants per plot. The dry method of planting was used in this concern. The other cultural practices of growing wheat were practiced. The studied raits.

1- Number of spikes / plant

2- Number of the kernels for

3- 1000-kernel weigh.

4- Grain yield per plant.

5- Biological yield

6- Harvest index.

\section{Statistical analysis:}

The data of all experiments were subjected to proper statisical analysis of varince according to Snedecor and Cochran (1967). The combined analysis across the two experiments (stress and normal irrigation) were performed according to Cochran and Cox (1957).For comparason between means. General (GCA) and specific (SCA) combining ability estimates were obtained by employing Griffing (1956) diallel cross analysis designated as method 2 model 1. 
Combining ability and genetic component analyses for wheat under

Table (1): The name pedigree and source of the parental varieties.

\begin{tabular}{|c|c|l|c|}
\hline NO & $\begin{array}{c}\text { genotypes } \\
\text { name }\end{array}$ & \multicolumn{1}{|c|}{ Pedigree } & Source \\
\hline 1 & Gemmeiza 7 & CMH74 A. 630/5x//Seri 82/3/Agent (Gemiza 7) & Egypt \\
\hline 2 & Gemmeiza 9 & ALD"S"/HUAC//CMH74A-630/SX & Egypt \\
\hline 3 & Gemmeiza 11 & $\begin{array}{l}\text { GOW"S"/KVS"S"I/7C/SERI82/3/GIZA168/SAKHA61 } \\
\text { GM-7892-2GM-1GM2GM-1GM-0GM }\end{array}$ & Egypt \\
\hline 4 & Gemmeiza 12 & $\begin{array}{l}\text { OTUS /3/SARA/THB/VEE } \\
\text { GMSS97YOO227S-5Y-010M-010Y-010M-2Y-1M-0Y-0GM }\end{array}$ & Egypt \\
\hline 5 & Sids 12 & $\begin{array}{l}\text { BUC//7C/ALD/5/MAYA74/ON//1160- } \\
\text { S47/3/BB/GLL/4/CHAT"S"/6/MAYA/VUL//CMH74A.630/4*SX. } \\
\text { SD7096-4SD-1SD-0SD }\end{array}$ & Egypt \\
\hline 6 & Misr 1 & $\begin{array}{l}\text { OASIS / SKAUZ I/4*BCN/3/2*PASTOR } \\
\text { CMSS00Y01881T-050M-030Y-030M-030WGY-33M-0Y-0S }\end{array}$ & Egypt \\
\hline 7 & Giza 171 & $\begin{array}{l}\text { Sakha 93 / Gemmeiza9 } \\
\text { GZ003-101-1GZ-1GZ-2GZ-0GZ }\end{array}$ & Egypt \\
\hline 8 & Giza 168 & MRI/BUG/SEPICM933046-8M-OY-OM-2Y-O3-OGZ. & \\
\hline
\end{tabular}

\section{RESULTS AND DISCUSSION}

Analysis of variances: Mean squares of different wheat genotypes for all studied characters in each environment and their combined data are presented in Table (2). Statistical analysis revealed significant of irrigation treatments for all studied characters, indicating that the two irrigation regimes behaved differently for these characters.

In addition, mean squares due to genotypes were highly significant for all traits, providing evidence for presence of large amount of genetic variability, which considered adequate for further biometrical assessment. Significant differences for all traits were found among the parents at both conditions and their combined.

Meanwhile, significant differences of crosses mean squares were detected for all characters, reflecting the diversity of the parents for these studied characters, and that these diversities could be transmitted to the progenies. Also, mean squares of parents vs. crosses showed significant differences for all traits, indicating the presence of hybrid vigor of the studied barley genotypes .

For all traits, mean squares of genotypes $x$ environments interactions were significant, indicating that genotypes responded differently to water regime for these traits and reflecting the possibility of selecting the most tolerant genotypes. Mean squares of parents $x$ environments, crosses $x$ environment and parent vs. crosses $x$ environment were highly significant for alltraits, except parent vs. crosses $x$ environment for 1000 kernel weight, revealing the performance of parents and most crosses were changed from environment to another.

Mean performances of the eight parents and their $F_{1}$ at stress and normal irrigation as well as their combined data are presented for all the studied characters in Table (3). It is clear that water stress condition decreased the mean number of spike per plant (NS/P), for the parents and hybrids. The highest NS/Pbelonged to parent 8 at the normal 


\begin{tabular}{|c|c|c|c|c|c|c|c|c|c|c|c|c|c|c|c|c|c|c|c|c|}
\hline \multirow{3}{*}{ 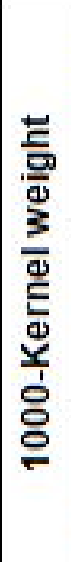 } & है & 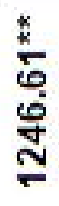 & $\stackrel{\text { * }}{\stackrel{*}{N}}$ & $\underset{\substack{*\\
}}{\stackrel{0}{\circ}}$ & \begin{tabular}{l}
$\stackrel{k}{*}$ \\
\multirow{2}{*}{} \\
$\stackrel{0}{0}$
\end{tabular} & $\begin{array}{l}\text { * } \\
\stackrel{0}{\circ} \\
\stackrel{0}{\circ}\end{array}$ & $\frac{{ }^{*}}{2}$ & 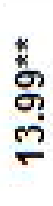 & 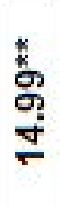 & 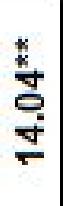 & $\bar{\emptyset}$ & लొ & 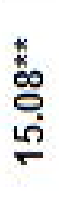 & 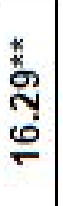 & $\underset{0}{\stackrel{*}{*}}$ & 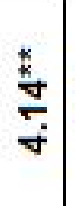 & $\stackrel{\infty}{\leftarrow}$ & $\stackrel{\text { }}{\sigma}$ & 巳̊ & 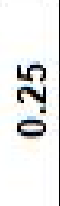 \\
\hline & in & & $\hat{m}$ & 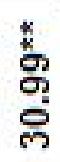 & 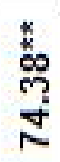 & 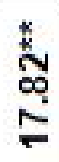 & 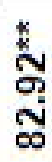 & & & & & $\stackrel{g}{\circ}$ & 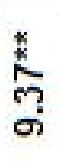 & 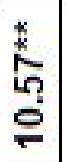 & & & $\stackrel{5}{\leftarrow}$ & $\begin{array}{l}\mathscr{I} \\
\stackrel{\infty}{0} \\
0\end{array}$ & & \\
\hline & $z$ & & 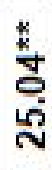 & $\frac{*}{5}$ & 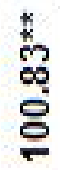 & $\begin{array}{l}\frac{k}{2} \\
\frac{0}{0} \\
\frac{m}{2}\end{array}$ & $\stackrel{n}{m}$ & & & & & $\ddot{\omega}$ & 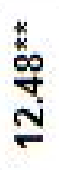 & $\begin{array}{l}* \\
\stackrel{*}{6} \\
\infty \\
\sigma \\
\sigma\end{array}$ & & & $\underset{乛}{~}$ & ָู & & \\
\hline
\end{tabular}

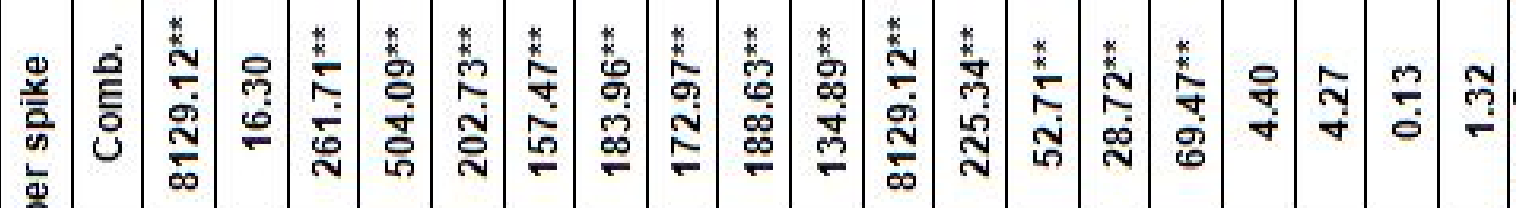

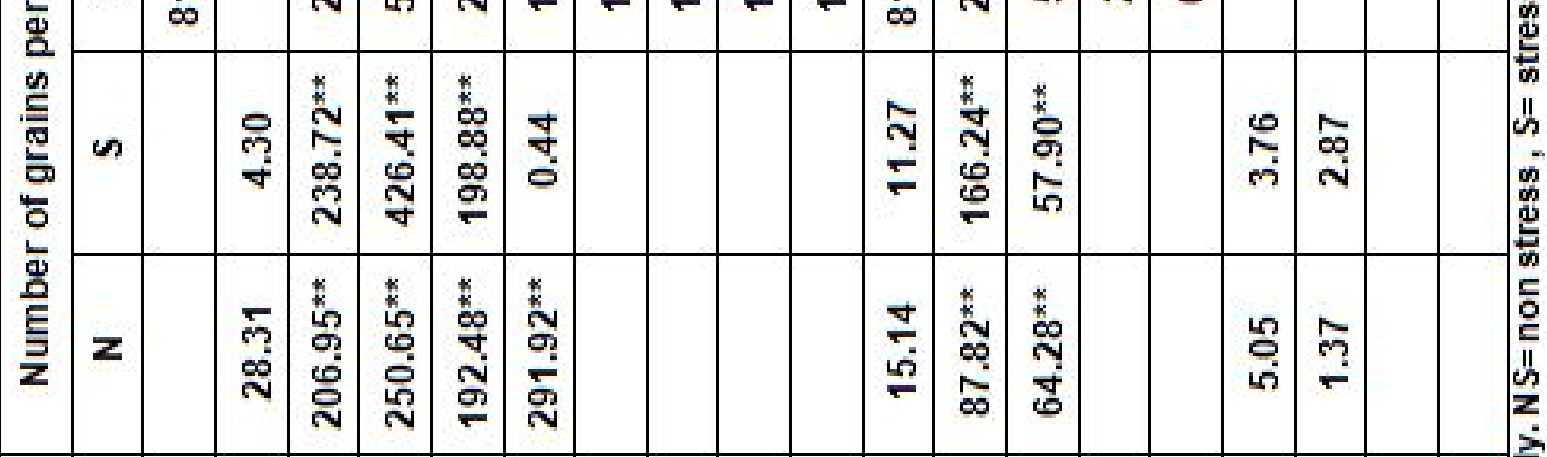

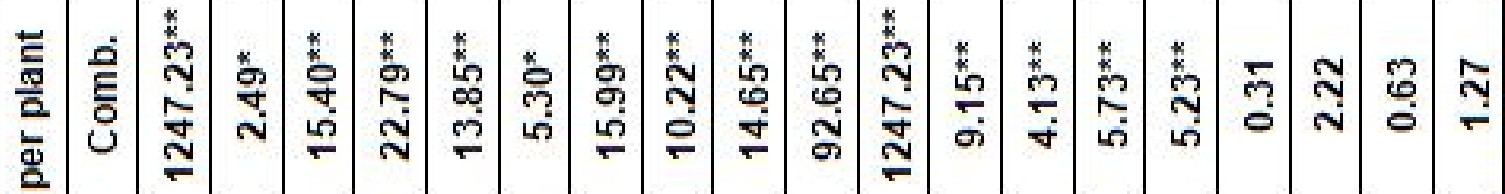

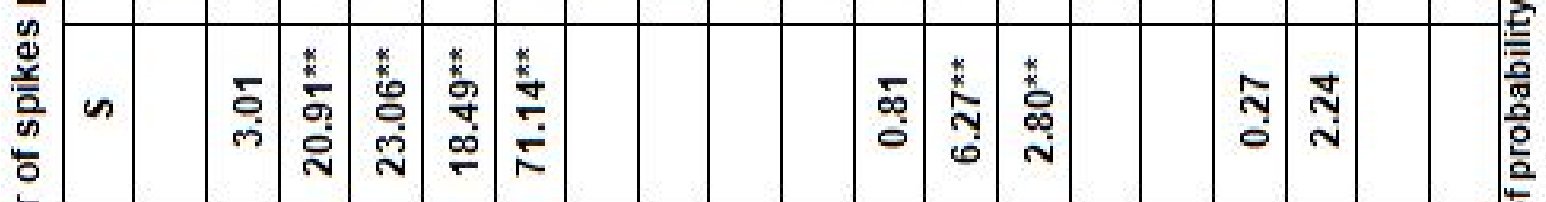

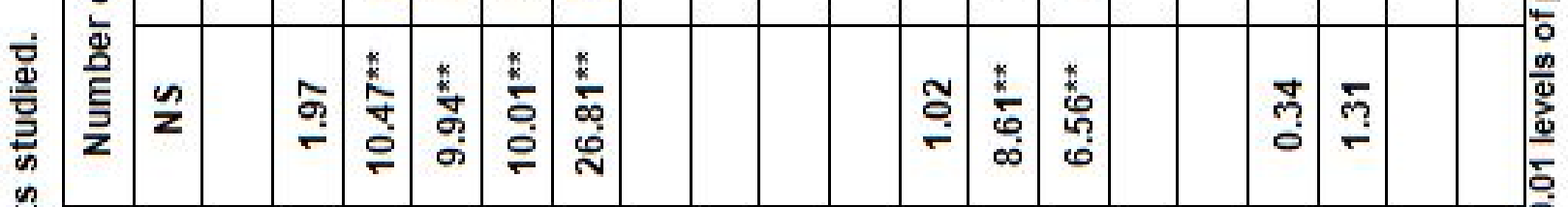

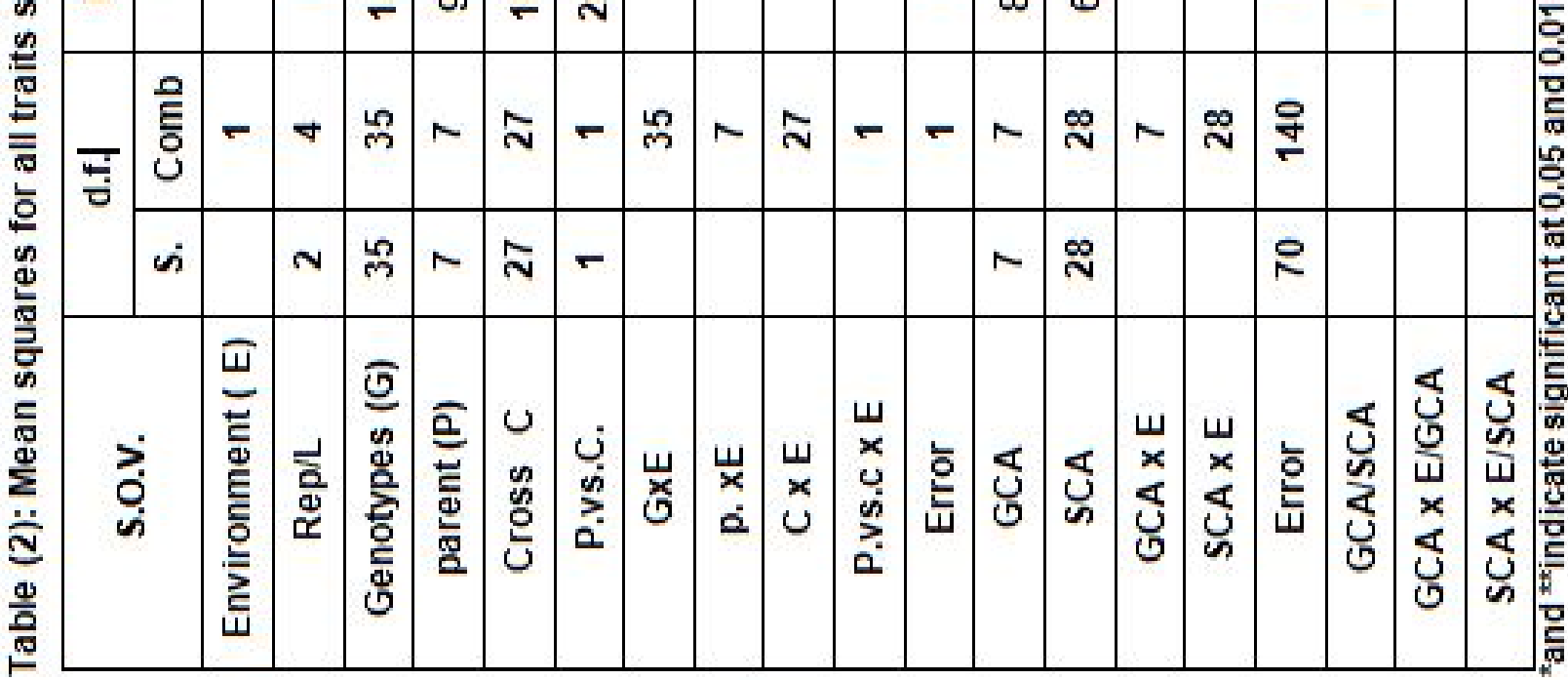




\begin{tabular}{|c|c|c|c|c|c|c|c|c|c|c|c|c|c|c|c|c|c|c|c|c|}
\hline \multirow{3}{*}{ 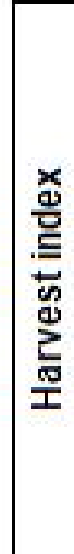 } & 官 & 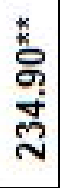 & ro & 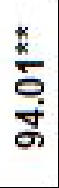 & $\underset{⿱ 乛}{\stackrel{0}{0}}$ & 葛 & $\begin{array}{l}\text { \% } \\
\text { ஸे }\end{array}$ & 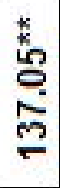 & 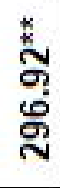 & $\frac{\text { * }}{\underset{*}{*}}$ & 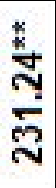 & $\begin{array}{l}\mathscr{M} \\
\stackrel{\infty}{\sim}\end{array}$ & 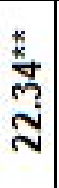 & 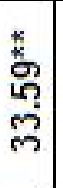 & 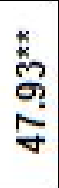 & 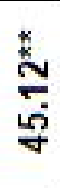 & 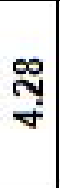 & $\ddot{0}$ & $\stackrel{n}{i}$ & 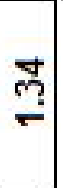 \\
\hline & in & & $\underset{\sim}{\infty}$ & 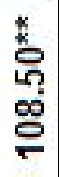 & m & $\underset{\text { ก }}{\stackrel{*}{*}}$ & స్ ది & & & & & 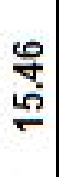 & 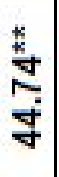 & 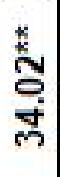 & & & $\underset{n}{\frac{1}{6}}$ & กั & & \\
\hline & z & & ָั้ & 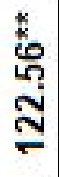 & 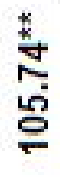 & $\begin{array}{l}\stackrel{*}{*} \\
\stackrel{2}{0} \\
\stackrel{2}{2}\end{array}$ & 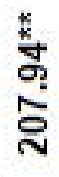 & & & & & ్ㅗ & 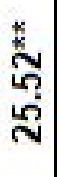 & 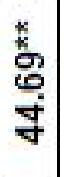 & & & ণे & ז? & & \\
\hline
\end{tabular}

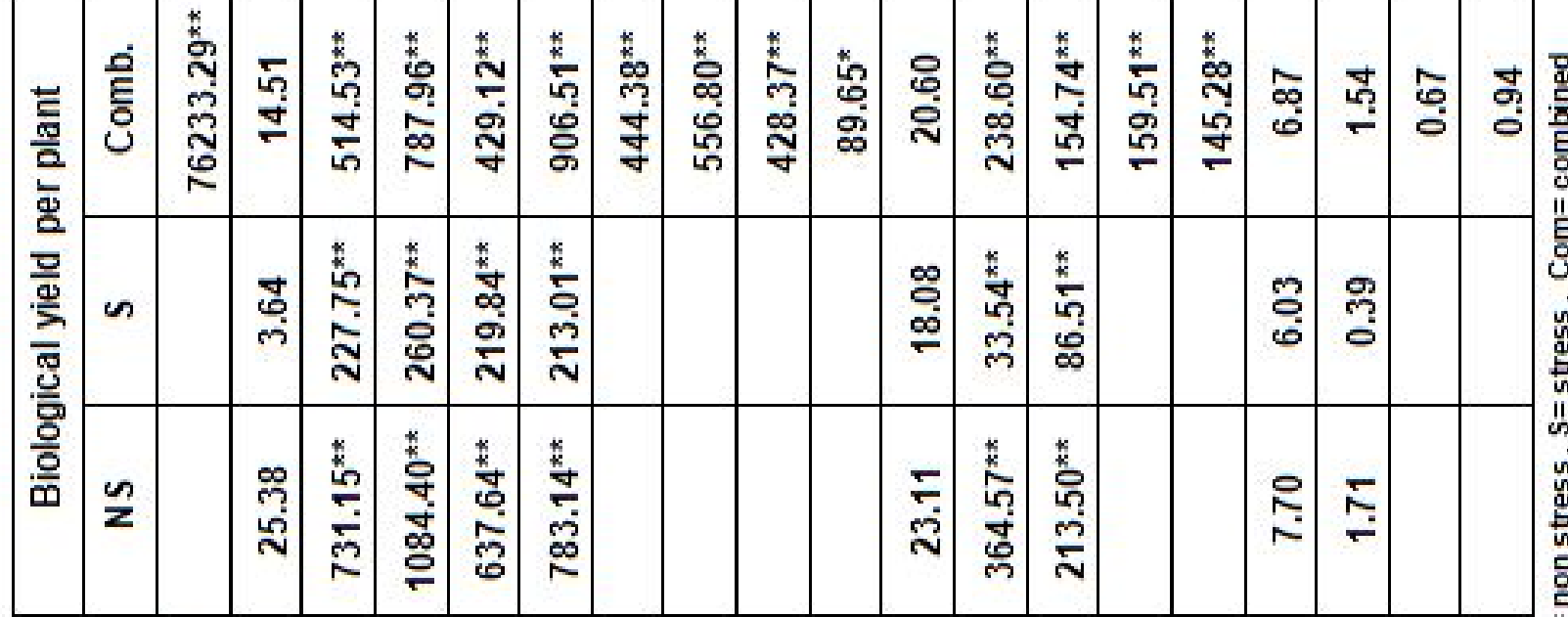

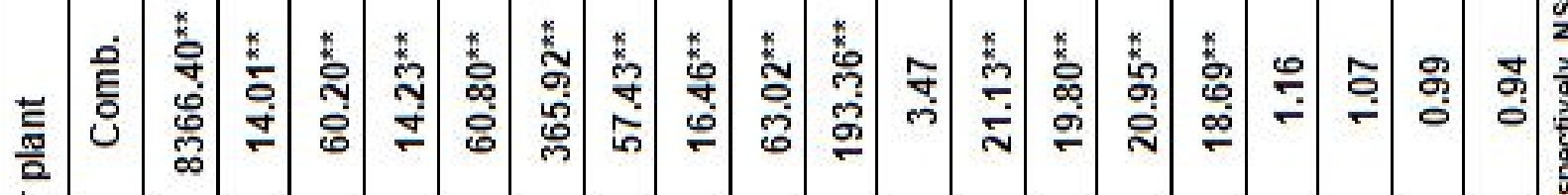

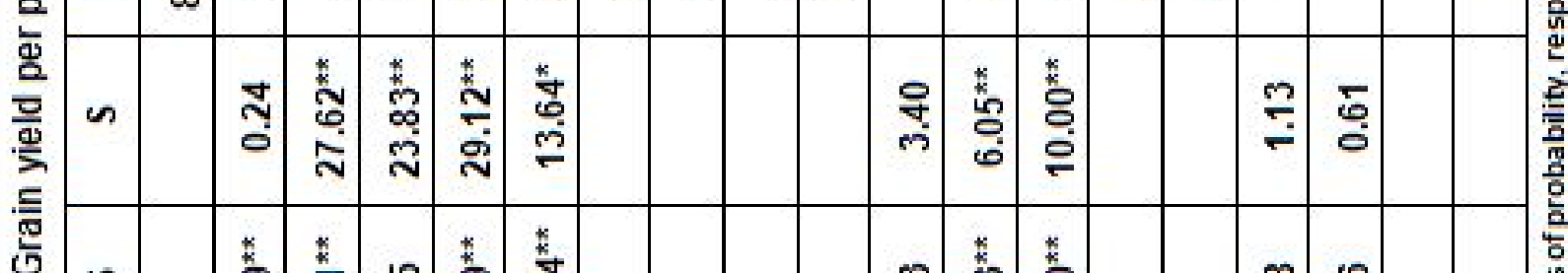

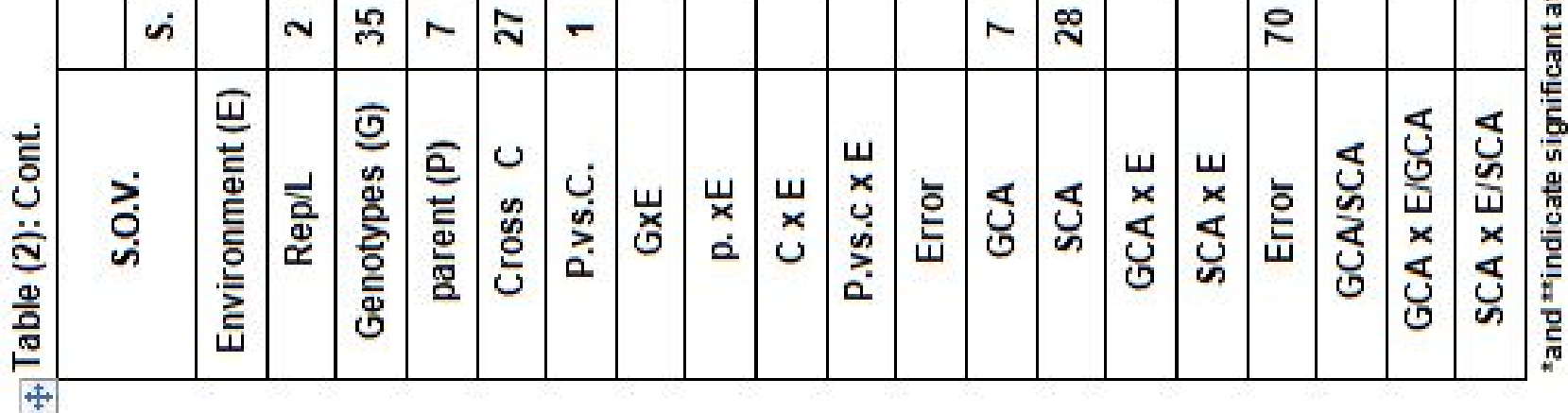

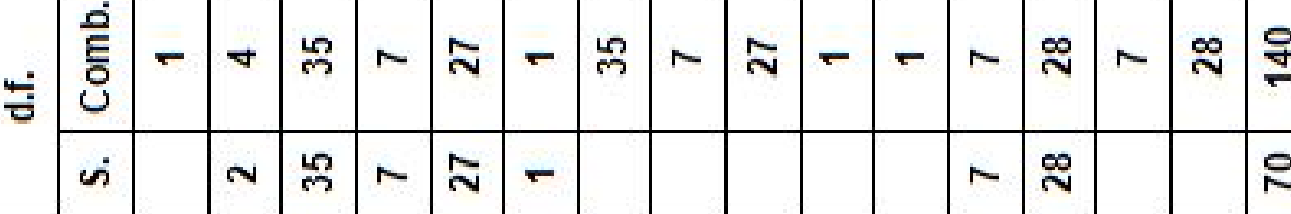


E.H. El-Seidy, et al.,

Table (3): The genotypes Mean performance for all traits studied.

\begin{tabular}{|c|c|c|c|c|c|c|c|c|c|}
\hline \multirow[b]{2}{*}{ Genotypes } & \multicolumn{3}{|c|}{$\begin{array}{c}\text { Number of spikes } \\
\text { per plant }\end{array}$} & \multicolumn{3}{|c|}{$\begin{array}{c}\text { Number of grains per } \\
\text { spike }\end{array}$} & \multicolumn{3}{|c|}{ 1000-Kernel weight } \\
\hline & NS & $\mathrm{s}$ & Comb. & NS & S & Comb. & NS & $\mathrm{s}$ & Comb \\
\hline$\left(P_{1}\right)$ Gemmeiza 7 & 12.00 & 9.97 & \begin{tabular}{|l||}
10.98 \\
\end{tabular} & 73.32 & 50.31 & 61.82 & 44.79 & 41.91 & 43.35 \\
\hline$\left(P_{2}\right)$ Gemmeiza 9 & 12.00 & 8.24 & \begin{tabular}{|l||}
10.12 \\
\end{tabular} & 67.90 & 53.57 & 60.73 & 43.67 & 38.86 & 41.26 \\
\hline$\left(P_{3}\right)$ Gemmeiza 11 & 6.90 & 6.50 & 6.70 & 85.35 & 48.65 & 67.00 & 43.23 & 36.60 & 39.91 \\
\hline$\left(P_{4}\right)$ Gemmeiza 12 & 8.83 & 10.68 & 9.76 & 66.90 & 55.32 & 61.11 & 32.62 & 29.89 & 31.26 \\
\hline$\left(P_{5}\right)$ Sids 12 & 7.70 & 5.70 & 6.70 & 85.60 & 79.25 & 82.43 & 48.63 & 36.61 & 42.62 \\
\hline$\left(P_{6}\right)$ Misr 1 & 11.70 & 10.54 & $\mid 11.12$ & 70.54 & 53.83 & 62.19 & 42.63 & 35.75 & 39.19 \\
\hline$\left(P_{7}\right)$ Giza 171 & 13.17 & 8.03 & \begin{tabular}{|l||}
10.60 \\
\end{tabular} & 75.95 & 73.88 & 74.92 & 43.40 & 40.84 & 42.12 \\
\hline$\left(P_{8}\right)$ Gemmeiza 7 & 14.79 & 8.58 & \begin{tabular}{|l||}
11.68 \\
\end{tabular} & 59.02 & 47.97 & 53.49 & 32.44 & 27.70 & 30.07 \\
\hline $1 \times 2$ & 13.47 & 4.80 & 9.13 & 73.80 & 43.50 & 58.65 & 40.19 & 40.70 & 40.44 \\
\hline $1 \times 3$ & 10.93 & 5.50 & 8.22 & 68.23 & 60.20 & 64.22 & 42.28 & 39.52 & 40.90 \\
\hline $1 \times 4$ & 9.80 & 7.87 & 8.83 & $\mid 70.83$ & 60.50 & 65.67 & 42.73 & 39.71 & 41.22 \\
\hline $1 \times 5$ & 10.93 & 8.63 & 9.78 & 60.67 & 52.48 & 56.57 & 39.93 & 34.53 & 37.23 \\
\hline $1 \times 6$ & 11.33 & 8.75 & \begin{tabular}{|l||}
10.04 \\
\end{tabular} & 53.63 & 43.42 & \begin{tabular}{|l|}
48.53 \\
\end{tabular} & 43.97 & 40.83 & 2.40 \\
\hline $1 \times 7$ & \begin{tabular}{|l|}
13.67 \\
\end{tabular} & 8.63 & \begin{tabular}{|l||}
11.15 \\
\end{tabular} & \begin{tabular}{|l||}
71.51 \\
\end{tabular} & 60.00 & \begin{tabular}{|l|}
65.76 \\
\end{tabular} & 41.59 & 35.21 & 38.40 \\
\hline $1 \times 8$ & 13.87 & 5.72 & 9.79 & 69.45 & 54.53 & \begin{tabular}{|l|}
61.99 \\
\end{tabular} & 41.13 & 38.02 & 39.57 \\
\hline $2 \times 3$ & 14.13 & 6.03 & \begin{tabular}{|l||}
10.08 \\
\end{tabular} & 56.67 & 54.37 & \begin{tabular}{|l|}
55.52 \\
\end{tabular} & 42.32 & 40.92 & 41.62 \\
\hline $2 \times 4$ & 18.22 & 7.22 & \begin{tabular}{|l||}
12.72 \\
\end{tabular} & \begin{tabular}{|l|l|}
62.90 \\
\end{tabular} & 58.20 & \begin{tabular}{|l|}
60.55 \\
\end{tabular} & 43.22 & 39.12 & 41.17 \\
\hline $2 \times 5$ & 15.81 & 6.85 & \begin{tabular}{|l||}
11.33 \\
\end{tabular} & 66.13 & 57.93 & 62.03 & 43.55 & 38.94 & 41.24 \\
\hline $2 \times 6$ & 11.44 & 9.73 & \begin{tabular}{|l||}
10.59 \\
\end{tabular} & \begin{tabular}{|l|l|}
65.09 \\
\end{tabular} & 59.30 & \begin{tabular}{|l|}
62.19 \\
\end{tabular} & 42.44 & 38.05 & 40.25 \\
\hline $2 \times 7$ & 15.93 & 6.92 & \begin{tabular}{|l||}
11.43 \\
\end{tabular} & \begin{tabular}{|l|l|}
63.35 \\
\end{tabular} & 69.10 & \begin{tabular}{|l|}
66.23 \\
\end{tabular} & 37.65 & 36.27 & 36.96 \\
\hline $2 \times 8$ & 12.90 & 6.65 & 9.78 & \begin{tabular}{|l||}
75.60 \\
\end{tabular} & 60.87 & \begin{tabular}{|l|}
68.23 \\
\end{tabular} & 44.75 & 36.64 & 40.70 \\
\hline $3 \times 4$ & 11.15 & 8.89 & \begin{tabular}{|l||}
10.02 \\
\end{tabular} & 75.43 & 53.64 & \begin{tabular}{|l|}
64.54 \\
\end{tabular} & 41.34 & 40.45 & 40.90 \\
\hline $3 \times 5$ & 12.54 & 6.27 & 9.41 & 61.63 & 79.77 & 70.70 & 45.28 & 40.82 & 43.05 \\
\hline $3 \times 6$ & 18.60 & 8.37 & \begin{tabular}{|l||}
13.48 \\
\end{tabular} & \begin{tabular}{|l||}
73.77 \\
\end{tabular} & 59.43 & \begin{tabular}{|l|}
66.60 \\
\end{tabular} & 43.44 & 37.27 & 40.36 \\
\hline $3 \times 7$ & 12.64 & 7.20 & 9.92 & \begin{tabular}{|l||}
73.10 \\
\end{tabular} & 59.57 & \begin{tabular}{|l|}
66.33 \\
\end{tabular} & 40.37 & 36.01 & 38.19 \\
\hline $3 \times 8$ & 14.13 & 4.13 & 9.13 & \begin{tabular}{|l|l|}
70.18 \\
\end{tabular} & 51.80 & \begin{tabular}{|l|}
60.99 \\
\end{tabular} & 47.15 & 40.66 & 43.91 \\
\hline $4 \times 5$ & \begin{tabular}{|l}
13.65 \\
\end{tabular} & 7.00 & \begin{tabular}{|l||}
10.33 \\
\end{tabular} & \begin{tabular}{|l||}
79.85 \\
\end{tabular} & 63.43 & \begin{tabular}{|l|}
71.64 \\
\end{tabular} & 40.67 & 37.00 & 38.83 \\
\hline $4 \times 6$ & 11.47 & 7.15 & 9.31 & 60.23 & 60.03 & \begin{tabular}{|l|}
60.13 \\
\end{tabular} & 42.39 & 34.91 & 38.65 \\
\hline $4 \times 7$ & 13.93 & 7.87 & \begin{tabular}{|l||}
10.90 \\
\end{tabular} & 61.80 & 53.99 & \begin{tabular}{|l|}
57.89 \\
\end{tabular} & 44.00 & 35.20 & 39.60 \\
\hline $4 \times 8$ & \begin{tabular}{|l|}
13.69 \\
\end{tabular} & 5.25 & 9.47 & 66.40 & 60.87 & \begin{tabular}{|l|}
63.63 \\
\end{tabular} & 43.32 & 41.43 & 42.38 \\
\hline $5 \times 6$ & 9.12 & 5.17 & 7.14 & $|72.99|$ & 49.32 & 61.15 & 42.22 & 38.03 & 40.12 \\
\hline $5 \times 7$ & 7.27 & 7.32 & 7.29 & \begin{tabular}{|l|l|}
89.90 \\
\end{tabular} & 63.57 & \begin{tabular}{|l|}
76.73 \\
\end{tabular} & 45.75 & 34.93 & 40.34 \\
\hline $5 \times 8$ & 10.73 & 5.25 & 7.99 & \begin{tabular}{|l||}
74.98 \\
\end{tabular} & 68.37 & \begin{tabular}{|l|}
71.67 \\
\end{tabular} & 43.47 & 41.73 & 42.60 \\
\hline $6 \times 7$ & 12.35 & 9.90 & \begin{tabular}{|l||}
11.13 \\
\end{tabular} & 82.77 & 41.98 & \begin{tabular}{|l|}
62.37 \\
\end{tabular} & 46.69 & 33.52 & 40.11 \\
\hline $6 \times 8$ & 11.70 & 11.13 & 11.42 & |63.27 & 50.53 & 56.90 & 42.54 & 36.94 & 39.74 \\
\hline $7 \times 8$ & 14.03 & \begin{tabular}{|l|}
11.08 \\
\end{tabular} & \begin{tabular}{|l||}
12.56 \\
\end{tabular} & \begin{tabular}{|l||} 
\\
\end{tabular} & 64.77 & \begin{tabular}{|l|}
67.97 \\
\end{tabular} & 42.86 & 40.18 & 41.52 \\
\hline mean of parent & 10.89 & 8.53 & 9.71 & 73.07 & 57.85 & 65.46 & 41.43 & 36.02 & 38.72 \\
\hline mean of cross & 12.84 & 7.33 & \begin{tabular}{|l||}
10.08 \\
\end{tabular} & 69.12 & 57.69 & \begin{tabular}{|l|}
63.41 \\
\end{tabular} & 42.76 & 38.13 & 40.44 \\
\hline mean of Genotype & 12.40 & 7.60 & 10.00 & 70.00 & 57.73 & 63.86 & 42.46 & 37.66 & 40.06 \\
\hline L.S.D 5\% & 1.64 & 1.47 & 1.53 & 6.32 & 5.45 & 5.82 & 3.11 & 3.02 & 3.02 \\
\hline L.S.D 1\% & 2.18 & 1.94 & 2.01 & 8.38 & 7.23 & 7.63 & 4.13 & 4.00 & 3.96 \\
\hline
\end{tabular}

*and **indicate significant at 0.05 and 0.01 levels of probability, respectively. NS= non stress , $\mathrm{S}=$ stress, $\mathrm{Com}=$ combined 
Combining ability and genetic component analyses for wheat under

Table (3): Cont.

\begin{tabular}{|c|c|c|c|c|c|c|c|c|c|}
\hline \multirow[b]{2}{*}{ Genotypes } & \multicolumn{3}{|c|}{ Grain yield per plant } & \multicolumn{3}{|c|}{$\begin{array}{c}\text { Biological yield per } \\
\text { plant }\end{array}$} & \multicolumn{3}{|c|}{ Harvest index } \\
\hline & NS & s & Comb. & NS & $\mathrm{s}$ & Comb. & NS & $\mathrm{s}$ & Comb. \\
\hline$\left(P_{1}\right)$ Gemmeiza 7 & 22.13 & 12.65 & 17.39 & \begin{tabular}{|l|}
72.70 \\
\end{tabular} & 40.63 & \begin{tabular}{|l|}
56.67 \\
\end{tabular} & 30.65 & 31.14 & 30.89 \\
\hline$\left(P_{2}\right)$ Gemmeiza 9 & 25.10 & 11.94 & 18.52 & 69.83 & 46.27 & 58.05 & 35.94 & 25.76 & 30.85 \\
\hline$\left(P_{3}\right)$ Gemmeiza 11 & 23.40 & 16.58 & 19.99 & 102.32 & 30.84 & 66.58 & 22.85 & 53.80 & 38.32 \\
\hline$\left(P_{4}\right)$ Gemmeiza 12 & 21.30 & 17.57 & 19.43 & 116.72 & 57.52 & 87.12 & 18.60 & 30.46 & 24.53 \\
\hline$\left(P_{5}\right)$ Sids 12 & 20.47 & 12.01 & 16.24 & \begin{tabular}{|l|}
66.23 \\
\end{tabular} & 38.43 & 52.33 & 30.89 & 32.47 & 31.68 \\
\hline$\left(P_{6}\right)$ Misr 1 & 20.70 & 14.73 & 17.72 & \begin{tabular}{|l|}
77.42 \\
\end{tabular} & 53.58 & 65.50 & 27.55 & 27.44 & 27.50 \\
\hline$\left(P_{7}\right)$ Giza 171 & 22.07 & 9.38 & 15.72 & \begin{tabular}{|l|}
79.54 \\
\end{tabular} & 55.47 & \begin{tabular}{|l|}
67.51 \\
\end{tabular} & 27.73 & 17.06 & 22.40 \\
\hline$\left(P_{8}\right)$ Gemmeiz & 21.93 & 10.99 & 16.46 & 61.67 & 42.41 & 52.04 & 35.56 & 25.94 & 30.75 \\
\hline $1 \times 2$ & 15.63 & 10.40 & 13.02 & \begin{tabular}{|l|l|}
87.04 \\
\end{tabular} & 45.07 & 66.06 & 17.98 & 23.26 & 20.62 \\
\hline $1 \times 3$ & 14.00 & 13.10 & .55 & \begin{tabular}{|l|}
65.65 \\
\end{tabular} & 45.59 & 55.62 & 21.64 & 28.75 & 25.20 \\
\hline $1 \times 4$ & 21.10 & 17.00 & 19.05 & \begin{tabular}{|l|}
69.00 \\
\end{tabular} & 59.21 & $\mid 64.10$ & 30.57 & 28.67 & 29.62 \\
\hline $1 \times 5$ & 21.70 & 10.43 & 16.07 & \begin{tabular}{|l|}
45.37 \\
\end{tabular} & 38.83 & \begin{tabular}{|l}
42.10 \\
\end{tabular} & 47.83 & 26.87 & 37.35 \\
\hline $1 \times 6$ & 29.07 & 15.83 & 22.45 & \begin{tabular}{|l|}
71.60 \\
\end{tabular} & 65.39 & \begin{tabular}{|l|}
68.49 \\
\end{tabular} & 40.59 & 24.25 & 32.42 \\
\hline $1 \times 7$ & 23.67 & 15.08 & 19.37 & \begin{tabular}{|l|}
89.37 \\
\end{tabular} & 55.00 & \begin{tabular}{|l}
72.18 \\
\end{tabular} & 26.50 & 27.41 & 26.95 \\
\hline $1 \times 8$ & 26.20 & 11.57 & 18.88 & \begin{tabular}{|l|}
79.65 \\
\end{tabular} & 42.43 & \begin{tabular}{|l|l}
61.04 \\
\end{tabular} & 32.93 & 27.32 & 30.13 \\
\hline $2 \times 3$ & 26.67 & 11.63 & 19.15 & \begin{tabular}{|l|}
85.30 \\
\end{tabular} & 44.73 & 65.02 & 31.25 & 26.12 & 28.69 \\
\hline $2 \times 4$ & 33.63 & 10.37 & 22.00 & \begin{tabular}{|l|}
92.07 \\
\end{tabular} & 45.72 & 68.89 & 36.56 & 22.92 & 29.74 \\
\hline $2 \times 5$ & 30.57 & 16.41 & 23.49 & 119.44 & 49.96 & \begin{tabular}{|l|l|}
84.70 \\
\end{tabular} & 25.73 & 32.64 & 29.19 \\
\hline $2 \times 6$ & 25.13 & 17.63 & 21.38 & 106.21 & 48.46 & 77.34 & 24.04 & 36.34 & 30.19 \\
\hline $2 \times 7$ & 30.53 & 17.75 & 24.14 & \begin{tabular}{|l|}
95.65 \\
\end{tabular} & 61.40 & 78.53 & 31.97 & 29.62 & 30.79 \\
\hline $2 \times 8$ & 27.17 & 13.72 & 20.44 & \begin{tabular}{|l|}
93.62 \\
\end{tabular} & 50.00 & \begin{tabular}{|l|}
71.81 \\
\end{tabular} & 29.36 & 27.22 & 28.29 \\
\hline $3 \times 4$ & 25.97 & 20.49 & 23.23 & \begin{tabular}{|l|}
78.43 \\
\end{tabular} & 63.85 & 71.14 & 33.29 & 32.49 & 32.89 \\
\hline $3 \times 5$ & 30.23 & 17.50 & 23.87 & \begin{tabular}{|l|}
91.61 \\
\end{tabular} & 56.93 & 74.27 & 33.26 & 30.63 & 31.95 \\
\hline $3 \times 6$ & 29.23 & 17.63 & 23.43 & \begin{tabular}{|l|}
95.37 \\
\end{tabular} & 55.33 & \begin{tabular}{|l|}
75.35 \\
\end{tabular} & 30.58 & 31.87 & 31.23 \\
\hline $3 \times 7$ & 25.77 & 16.50 & 21.13 & \begin{tabular}{|l|}
95.17 \\
\end{tabular} & 48.40 & \begin{tabular}{|l|l|}
71.78 \\
\end{tabular} & 27.14 & 34.20 & 30.67 \\
\hline $3 \times 8$ & 26.27 & 10.40 & 18.33 & \begin{tabular}{|l|}
76.30 \\
\end{tabular} & 52.40 & \begin{tabular}{|l|l}
64.35 \\
\end{tabular} & 34.38 & 19.87 & 27.13 \\
\hline $4 \times 5$ & 37.03 & 12.13 & 24.58 & 102.30 & 48.97 & \begin{tabular}{|l|l}
75.63 \\
\end{tabular} & 36.20 & 25.22 & 30.71 \\
\hline $4 \times 6$ & 29.40 & 11.74 & 20.57 & \begin{tabular}{|l|}
81.23 \\
\end{tabular} & 39.52 & \begin{tabular}{|l|}
60.38 \\
\end{tabular} & 36.21 & 29.62 & 32.91 \\
\hline $4 \times 7$ & 27.43 & 11.81 & 19.62 & \begin{tabular}{|l|}
89.52 \\
\end{tabular} & 38.82 & 64.17 & 30.68 & 30.39 & 30.53 \\
\hline $4 \times 8$ & 38.63 & 9.97 & 24.30 & 106.61 & 31.17 & \begin{tabular}{|l|}
68.89 \\
\end{tabular} & 36.37 & 32.25 & 34.31 \\
\hline $5 \times 6$ & 23.37 & 9.93 & 16.65 & \begin{tabular}{|l|}
88.62 \\
\end{tabular} & 41.83 & \begin{tabular}{|l|l}
65.23 \\
\end{tabular} & 26.80 & 23.91 & 25.35 \\
\hline $5 \times 7$ & 29.73 & 12.87 & 21.30 & \begin{tabular}{|l|}
84.59 \\
\end{tabular} & 49.05 & \begin{tabular}{|l|l}
66.82 \\
\end{tabular} & 35.23 & 26.54 & 30.88 \\
\hline $5 \times 8$ & 31.60 & 15.75 & 23.68 & \begin{tabular}{|l|}
81.58 \\
\end{tabular} & 41.28 & 61.43 & 38.53 & 38.18 & 38.36 \\
\hline $6 \times 7$ & 37.13 & 12.04 & 24.59 & \begin{tabular}{|l|}
85.55 \\
\end{tabular} & 38.43 & \begin{tabular}{|l|l|}
61.99 \\
\end{tabular} & 43.42 & 31.25 & 37.34 \\
\hline $6 \times 8$ & 25.53 & 16.68 & 21.11 & \begin{tabular}{|l|}
82.73 \\
\end{tabular} & 57.39 & \begin{tabular}{|l|l|}
70.06 \\
\end{tabular} & 30.99 & 29.09 & 30.04 \\
\hline $7 \times 8$ & 28.83 & 18.02 & 23.43 & $\mid 104.33$ & 57.43 & 80.88 & 27.62 & 31.41 & 29.52 \\
\hline mean of parent & 22.14 & 13.23 & 17.68 & $\mid$\begin{tabular}{||l}
80.81 \\
\end{tabular} & 45.64 & $\mid$\begin{tabular}{||l} 
\\
63.22
\end{tabular} & 28.72 & 30.51 & 29.62 \\
\hline mean of cross & 27.54 & 14.09 & 20.81 & 87.28 & 49.02 & 68.15 & 32.06 & 28.87 & 30.46 \\
\hline mean of Genotype & 26.34 & 13.90 & 20.12 & 85.84 & 48.27 & 67.06 & 31.32 & 29.23 & 30.28 \\
\hline L.S.D 5\% & 3.05 & 2.99 & 2.98 & 7.81 & 6.91 & 7.26 & 5.19 & 6.39 & 5.73 \\
\hline L.S.D 1\% & 4.05 & 3.97 & 3.91 & 10.35 & 9.16 & 9.52 & 6.88 & 8.47 & 7.52 \\
\hline
\end{tabular}

*and **indicate significant at 0.05 and 0.01 levels of probability, respectively. NS= non stress , $\mathrm{S}=$ stress, Com $=$ combined 
irrigation as well as the combined analysis. While, parent 5 showed the smallest NS/P at the normal irrigation as well as the combined analysis. Abd ElAty and El-Borhamy (2007) found significant differences among wheat genotypes in NS/P. The highest NS/P was obtained from the following crosses; $p_{3} x$ $p_{6}$ under normal environment and $p_{6} x$ $p_{8}$ at the drought condition.

With regard to number of kernels per spike (NK/S), the parents 5 showed the highest values at the two conditions and their combined while, parent 8 revealed lowest number of NK/S at the drought conditions. Also crosses; $p_{5} \times p_{7}$ showed the highest values at the normal condition and combined analysis and $p_{3}$ $x p_{5}$ gave the highest value at the drought condition. While, $p_{6} \times p_{7}$ showed the lowest values at the drought condition.

With regard to the parents for 1000 kernel weight, the heaviest were obtained from parent 5 under the normal condition and parent lunder drought condition as well as the combined analysis. The heaviest 1000-kernel weight of wheat hybrids were obtained from $p_{3} \times p_{8}$ at normal irrigation and $p_{5} \times p_{8}$ under the drought condition. While, the lightest 1000- kernel weight of wheat crosses were relative to $p_{6} \times p_{7}$.

As a result of water stress condition, the average of grain yield/plant (GYIP) for parents and their hybrids was decreased. Several investigators reported that drought stress reduced photosynthesis and translocation rates and increased respiration, which reduced available assimilates for grain filling and finally decreased GYIP. Abd El-Aty and EIBorhamy (2007) found similar results. The highest GY/P were showed by parent 2 under the normal condition and parent 4under drought as well as the combined analysis. While, the lowest GY/P was obtained by parent 7 under drought data. The hybrids, $p_{4} \times p_{8}$, at normal condition, $p_{3} \times p_{4}$ at drought condition and $p_{6} \times p_{7}$ at the combined analysisyielded more than the other crosses. While, $p_{5} \times p_{6}$ gave the lowest values under drought condition. The highest GY/P of these parents and crosses could be attributed to the highest GYIP of $p_{3}$ and $p_{4}$, which may possessed the genes controlling in GYIP.

With regard to number of biological yield/plant, the parents 4 showed the highest values at the two conditions and their combined while, parent 3 revealed lowest number of biological yield/plant at the drought conditions. Also crosses; $p_{2}$ $x p_{5}$ showed the highest values at the normal condition and combined analysis. While, $p_{4} \times p_{8}$ showed the lowest values at the drought condition.

For harvest index, the parents 2 showed the highest values at the normal condition and parent 3 under drought condition and the combined analysis. while, parent 7 revealed lowest number of harvest index at the drought condition. Also crosses; $p_{5} \times p_{8}$ showed the highest values at the drought condition and combined analysis. While, $p_{3} \times p_{8}$ showed the lowest values at the drought condition.

Mean performance of F1 crosses for all studied traits in each treatment as well as combined data are presented in Table (3). Regarding number of spikes plant-1, the highest mean values in normal irrigation (N/S) (18.6), were detected for the cross P3 x P6. And P6xP8 in(S) data (11.13).

For No. of grains/ spike, the cross P5 $X$ P7 gave the highest values (89.9), (76.73), under (N/S) condition and combined analysis, and the cross P3 $x$ P5 gave the highest values (79.77) in(S).

The parental combination P3 $x$ P8 gave the highest mean values for 1000 - 
kernel weight in (N/S) treatment, and, gave higher value the cross $\mathrm{P} 5 \times \mathrm{P8}(\mathrm{S})$.

For grain yield/ plant the cross $\mathrm{P} 4 \times \mathrm{P8}$ gave the highest values $(38.63 \mathrm{~g})$ under normal condition. However, the highest mean values for grain yield/ plant (20.49) were detected by P3 $x$ P4. Moreover the cross P6 $\times$ P7 exhibited the heavier grain yield plant in the combined analysis being 24.59 .

The highest mean values for biological yield I plant were detected by the cross P2 $\times$ P5 under normal condition and combined analysis (119.44g), (84.70).

For harvest index, the cross P5 $x$ P8 gave the highest values (38.18), (38.36), under drought stress and combined analysis, and the cross P1 $\times$ P5 gave the highest values (47.83) in normal environment.

Combining ability analysis: Combining ability implies the capacity of parent to produce good progenies when crossed with the other parent.

Analysis of variance for combining ability as out lined by Griffing (1956) method 2 model 1 in each environment as well as their combined for all the studied traits in Table (2).

The results indicate that mean squares of general combining ability (GCA) and specific combining ability (SCA) were highly significant for all the studied traits under the two environments and their combined.

The ratios between GCA and SCA exceeded the unity for all studied traits except for straw yield and harvest index at normal irrigation and grain yield/ plant, biological yield/ plant1000-kernel weight, straw yield, in the drought condition and straw yield at combined analysis, revealing that additive and additive $x$ additive types of gene action are more important than non-additive gene action in controlling these traits. These results were coincident with those reported by Gomaa et al (2014) and El Hosary et al. (2015).

General combining ability effects: Estimates of GCA ( $\hat{g} \mathbf{i})$ effects of all wheat parental genotypes for each trait in combined data are presented in Table (4). Such effects are being used to compare the average performance of each parent with the other and facilitate selection of parents for further improvement to drought tolerance.

Results indicated that the parent P1 gave significant and positive gi effects for1000-kernel weight in drought condition and the combined analysis. The parental $P_{2}$ exhibited significant and positive gi effects for no. of spikes per plant and biological yield per plant in normal irrigation environment and the combined analysis and1000-kernel weight under drought and combined analysis. The parent $P_{3}$ exhibited significant and positive ĝi effects for grain yield per plant under drought environment condition. Also, this parent considered the best combiner for biological yield per plant, No. of grains per spike at normal irrigation treatment and the combined analysis. The parent $P_{4}$ expressed significant and positive gi $i$ effects for grain yield per plant and biological yield per plant in normal environment and the combined analysis. The parent $P_{5}$ expressed significant and positive ĝi effects for number of grains per spike in both and across environments, 1000-kernels wight, harvest index in normal and combined analysis. The parent $p_{6}$ expressed significant and positive ĝi effects for No. of spikes I plant, biological yield I plant under drought and combined analysis. The parent $\mathrm{P}_{7}$ exhibited significant and negative gi effects for No. of spikes I plant, biological yield $I$ plant and number of grains per spike both and across environments, grain yield $I$ plant in normal and combined analysis. The 
E.H. El-Seidy, et al.,

parent $P_{8}$ seemed to be the best general combiner for No. of spikes I plant and harvest index under normal environment as well as the combined analysis.

Table (4): Estimates of general combining ability effects for all studies treats.

\begin{tabular}{|c|c|c|c|c|c|c|c|c|c|}
\hline \multirow[b]{2}{*}{ Parents } & \multicolumn{3}{|c|}{\begin{tabular}{|c|}
$\begin{array}{c}\text { Number of spikes per } \\
\text { plant }\end{array}$ \\
\end{tabular}} & \multicolumn{3}{|c|}{$\begin{array}{c}\begin{array}{c}\text { Number of grains per } \\
\text { spike }\end{array} \\
\end{array}$} & \multicolumn{3}{|c|}{ 1000-Kernel weight } \\
\hline & NS & $\mathbf{S}$ & Comb. & NS & $\mathbf{S}$ & Comb. & NS & $\mathbf{S}$ & Comb. \\
\hline$\left(P_{1}\right)$ Gemmeiza 7 & $-0.36^{*}$ & 0.15 & -0.11 & $-1.52^{*}$ & $-4.43^{* *}$ & $-2.98^{* *}$ & -0.08 & $1.34^{\star *}$ & $0.63^{* *}$ \\
\hline$\left(P_{2}\right)$ Gemmeiza 9 & $1.43^{* *}$ & $-0.37^{*}$ & $0.53^{\star *}$ & $-3.06^{* *}$ & -0.92 & $-1.99^{* *}$ & -0.07 & $0.94^{\star *}$ & $0.44^{* *}$ \\
\hline$\left(P_{3}\right)$ Gemmeiza 11 & $-0.37^{*}$ & $-0.90^{\star *}$ & $-0.63^{* *}$ & $1.97^{\star *}$ & -0.35 & $0.81^{* *}$ & 0.65 & $0.99^{* \star}$ & $0.82^{\star *}$ \\
\hline$\left(P_{4}\right)$ Gemmeiza 12 & -0.21 & $0.42 * *$ & 0.11 & $-1.87^{* *}$ & 0.17 & $-0.85^{* *}$ & $-1.93^{* *}$ & $-1.13^{* *}$ & $-1.53^{* *}$ \\
\hline$\left(P_{5}\right)$ Sids 12 & $-1.62^{\star *}$ & $-1.05^{\star *}$ & $-1.33^{* *}$ & $4.74^{\star *}$ & $7.38^{\star *}$ & $6.06^{* *}$ & $1.60^{* *}$ & 0.03 & $0.81^{* *}$ \\
\hline$\left(P_{6}\right)$ Misr 1 & -0.22 & $1.29 * *$ & $0.53^{* *}$ & $-1.72^{\star}$ & $-4.79^{* *}$ & $-3.25^{* *}$ & $0.68^{*}$ & $-0.79^{*}$ & -0.05 \\
\hline$\left(P_{7}\right)$ Giza 171 & $0.45^{*}$ & $0.66 * *$ & $0.56^{\star *}$ & $3.55^{\star *}$ & $4.12^{\text {** }}$ & $3.83^{* *}$ & 0.35 & -0.59 & -0.12 \\
\hline$\left(P_{8}\right)$ Gemmeiza 7 & $0.90^{* *}$ & -0.20 & $0.35^{* *}$ & $-2.09 * *$ & $-1.19 *$ & $-1.64^{* *}$ & $-1.21^{* *}$ & $-0.79^{*}$ & $-1.00^{* *}$ \\
\hline L.S.D(0.05) gi & 0.34 & 0.31 & 012 & 1.32 & 1.14 & 0.48 & 0.65 & 0.63 & 0.25 \\
\hline L.S.D(0.01) gi & 0.46 & 0.41 & 0.17 & 1.75 & 1.51 & 0.64 & 0.86 & 0.84 & 0.33 \\
\hline L.S.D(0.05) gi-gj & 0.52 & 0.46 & 0.24 & 2.00 & 1.73 & 0.92 & 0.98 & 0.96 & 0.48 \\
\hline L.S.D(0.01) gi-gj & 0.69 & 0.62 & 0.32 & 2.65 & 2.29 & 1.21 & 1.31 & 1.27 & 0.63 \\
\hline
\end{tabular}

*and **indicate significant at 0.05 and 0.01 levels of probability, respectively. NS= non stress, $\mathrm{S}=$ stress , Com= combined

Table (4): con.

\begin{tabular}{|c|c|c|c|c|c|c|c|c|c|}
\hline \multirow[b]{2}{*}{ Parents } & \multicolumn{3}{|c|}{ Grain yield per plant } & \multicolumn{3}{|c|}{$\begin{array}{l}\text { Biological yield per } \\
\text { plant }\end{array}$} & \multicolumn{3}{|c|}{ Harvest index } \\
\hline & NS & S & Comb. & NS & S & Comb. & NS & S & Comb. \\
\hline$\left(P_{1}\right)$ Gemmeiza 7 & $-4.15^{\star *}$ & $-0.63^{*}$ & $-2.39 * *$ & $-11.95 * *$ & -0.17 & $-6.06^{* *}$ & -0.25 & $-1.43^{*}$ & $-0.84^{\star *}$ \\
\hline$\left(P_{2}\right)$ Gemmeiza 9 & 0.24 & -0.33 & -0.04 & $4.64^{\star \star}$ & 0.34 & $2.49^{* *}$ & $-1.31^{*}$ & $-1.34^{*}$ & $-1.33^{\star *}$ \\
\hline$\left(P_{3}\right)$ Gemmeiza 11 & $-1.22^{* *}$ & $1.53^{* *}$ & 0.16 & $1.99^{*}$ & -0.55 & $0.72^{*}$ & $-2.46^{* *}$ & $4.84^{* *}$ & $1.19 * *$ \\
\hline$\left(P_{4}\right)$ Gemmeiza 12 & $1.87^{* *}$ & 0.36 & $1.11 * *$ & $8.00^{* *}$ & 0.79 & $4.39 * *$ & -0.48 & -0.06 & -0.27 \\
\hline$\left(P_{5}\right)$ Sids 12 & $0.81^{*}$ & -0.60 & 0.10 & $-2.66^{* *}$ & $-3.07^{* *}$ & $-2.87^{* *}$ & $2.35^{\star *}$ & 0.58 & $1.47^{* *}$ \\
\hline$\left(P_{6}\right)$ Misr 1 & 0.32 & 0.59 & $0.45^{\star *}$ & -0.64 & $1.91^{*}$ & $0.63^{*}$ & 0.59 & -0.19 & 0.20 \\
\hline$\left(P_{7}\right)$ Giza 171 & $1.02^{* *}$ & -0.22 & $0.40 * *$ & $3.07^{* *}$ & $2.50 * *$ & 2.79 ** & -0.38 & $-1.81 * *$ & $-1.10^{* *}$ \\
\hline$\left(P_{8}\right)$ Gemmeiza 7 & $1.10^{* *}$ & $-0.70^{*}$ & 0.20 & $-2.44^{\star *}$ & $-1.75^{\star}$ & $-2.10^{* *}$ & $1.95^{\star *}$ & -0.59 & $0.68^{* *}$ \\
\hline L.S.D(0.05) gi & 0.64 & 0.63 & 0.25 & 1.63 & 1.45 & 0.61 & 1.09 & 1.34 & 0.48 \\
\hline L.S.D(0.01) gi & 0.85 & 0.83 & 0.33 & 2.17 & 1.92 & 0.79 & 1.44 & 1.77 & 0.63 \\
\hline L.S.D(0.05) gi-gj & 0.97 & 0.95 & 0.47 & 2.47 & 2.18 & 1.15 & 1.64 & 2.02 & 0.91 \\
\hline L.S.D(0.01) gi-gj & 1.28 & 1.26 & 0.62 & 3.28 & 2.90 & 1.51 & 2.18 & 2.68 & 1.19 \\
\hline
\end{tabular}

*and **indicate significant at 0.05 and 0.01 levels of probability, respectively. NS= non stress , $\mathrm{S}=$ stress , Com $=$ combined 
Menoufia J. Plant Prod., Vol. 3 December (2018): 367 - 381

Specific combining ability effects (Sij):
SCA (Sij) of the parental combinations computed for seven traits in combined analysis are presented in Table (5).

Table (5): Estimates of specific combining ability effects for all studied traits .

\begin{tabular}{|c|c|c|c|c|c|c|c|c|c|}
\hline \multirow[b]{2}{*}{ crosses } & \multicolumn{3}{|c|}{$\begin{array}{l}\text { Number of spikes } \\
\text { per plant }\end{array}$} & \multicolumn{3}{|c|}{$\begin{array}{l}\text { Number of grains per } \\
\text { spike }\end{array}$} & \multicolumn{3}{|c|}{ 1000-Kernel weight } \\
\hline & NS & $\mathbf{s}$ & Comb. & NS & $\mathbf{s}$ & Comb. & NS & $\mathrm{s}$ & Comb. \\
\hline P1xP2 & 0.00 & $-2.58^{\star *}$ & $-1.29 * *$ & $8.39 * *$ & $-8.88^{* *}$ & -0.25 & $-2.13^{*}$ & 0.75 & -0.69 \\
\hline P1xP3 & -0.74 & $-1.34^{* *}$ & $-1.04^{* *}$ & -2.22 & $7.25^{\star *}$ & 2.52 & -0.75 & -0.47 & -0.61 \\
\hline P1xP4 & $-2.04^{* *}$ & -0.30 & $-1.17^{* *}$ & $4.23^{*}$ & $7.03^{* *}$ & $5.63^{* *}$ & $2.27^{*}$ & 1.84 & $2.05^{\star *}$ \\
\hline P1xP5 & 0.51 & $1.94^{\star *}$ & $1.22 * *$ & $-12.55^{* *}$ & $-8.20 * *$ & $-10.37 * *$ & $-4.05^{\star *}$ & $-4.49^{* *}$ & $-4.27^{* *}$ \\
\hline P1xP6 & -0.49 & -0.28 & -0.38 & $-13.13^{* *}$ & $-5.09 * *$ & $-9.11^{* *}$ & 0.90 & $2.62^{\star \star}$ & $1.76^{*}$ \\
\hline P1xP7 & $1.17^{*}$ & 0.23 & $0.70^{*}$ & -0.52 & 2.59 & 1.03 & -1.15 & $-3.20^{* *}$ & $-2.18^{\star *}$ \\
\hline P1xP8 & 0.93 & $-1.82^{* *}$ & -0.45 & 3.07 & 2.42 & $2.75^{*}$ & -0.05 & -0.19 & -0.12 \\
\hline P2xP3 & 0.67 & -0.30 & 0.19 & $-12.24^{* *}$ & -2.10 & $-7.17^{* *}$ & -0.72 & 1.33 & 0.30 \\
\hline P2xP4 & $4.59^{* *}$ & -0.43 & $2.08^{* *}$ & -2.16 & 1.21 & -0.48 & $2.76^{\star *}$ & 1.65 & $2.20 * *$ \\
\hline P2xP5 & $3.60^{* *}$ & 0.67 & $2.13^{* *}$ & $-5.54^{* *}$ & $-6.26^{* *}$ & $-5.90 * *$ & -0.44 & 0.31 & -0.07 \\
\hline P2xP6 & $-2.17 * \star$ & $1.21^{*}$ & -0.48 & -0.13 & $7.28 * *$ & $3.57^{* *}$ & -0.63 & 0.24 & -0.20 \\
\hline P2xP7 & $1.65^{* *}$ & $-0.97^{*}$ & 0.34 & $-7.13^{* *}$ & $8.17^{* *}$ & 0.52 & $-5.10^{\star *}$ & -1.74 & $-3.42^{* *}$ \\
\hline P2xP8 & $\mid-1.83^{* *}$ & -0.38 & $-1.10 * *$ & $10.76^{* *}$ & $5.24^{\star *}$ & $8.00^{* *}$ & $3.57^{* *}$ & -1.17 & 1.20 \\
\hline P3xP4 & -0.68 & $1.77^{* *}$ & 55 & $5.33^{*}$ & $-3.91^{*}$ & 0.71 & 0.15 & $2.93^{\star *}$ & $1.54^{*}$ \\
\hline P3xP5 & $2.13^{* \star}$ & 0.62 & $1.37^{* *}$ & $-15.08^{* *}$ & $15.00^{* *}$ & -0.04 & 0.57 & $2.14^{*}$ & 1.36 \\
\hline P3xP6 & $6.79^{* \star}$ & 0.38 & $3.58^{* *}$ & 3.51 & $6.84^{\star *}$ & $5.17^{* *}$ & -0.35 & -0.59 & -0.47 \\
\hline P3xP7 & 0.15 & -0.16 & 0.00 & -2.42 & -1.93 & -2.18 & -3.09 ** & $-2.04^{*}$ & $-2.57^{* *}$ \\
\hline P3xP8 & $1.20 *$ & $-2.36^{* *}$ & -0.58 & 0.30 & $-4.39 *$ & -2.05 & $5.25^{* *}$ & $2.80^{\star *}$ & $4.03^{* *}$ \\
\hline P4xP5 & $3.07^{* \star}$ & 0.03 & $1.55^{\star \star}$ & $6.99 * *$ & -1.85 & 2.57 & -1.47 & 0.44 & -0.51 \\
\hline P4xP6 & -0.50 & $-2.16^{\star *}$ & $-1.33^{\star *}$ & $-6.18^{\star *}$ & $6.92^{\star *}$ & 0.37 & 1.18 & -0.83 & 0.17 \\
\hline P4xP7 & $1.28^{*}$ & -0.81 & 0.23 & $-9.88^{* *}$ & $-8.03^{* *}$ & $-8.95^{\star *}$ & $3.11^{* *}$ & -0.73 & 1.19 \\
\hline P4xP8 & 0.59 & $-2.57^{* *}$ & $-0.99 * *$ & 0.36 & $4.15^{*}$ & 2.26 & $3.99^{* *}$ & $5.70^{* *}$ & $4.84^{\star *}$ \\
\hline P5xP6 & $\mid-1.45^{* *}$ & $-2.67^{* *}$ & $-2.06^{* *}$ & -0.03 & $-11.00^{* *}$ & $-5.52^{\star *}$ & $-2.52^{*}$ & 1.13 & -0.70 \\
\hline P5xP7 & $\mid-3.97^{* \star}$ & 0.11 & $-1.93^{* *}$ & $11.61^{* *}$ & $-5.66^{* *}$ & $2.98^{*}$ & 1.34 & $-2.16^{*}$ & -0.41 \\
\hline P5xP8 & -0.95 & \begin{tabular}{|l|}
$-1.09^{*}$ \\
\end{tabular} & $-1.02^{* *}$ & 2.33 & $4.45^{*}$ & 3.39* & 0.62 & $4.84^{* *}$ & $2.73^{* *}$ \\
\hline P6xP7 & -0.28 & 0.35 & 0.03 & $10.93^{* *}$ & $-15.08^{* \star}$ & -2.07 & $3.20^{* *}$ & $-2.76^{\star *}$ & 0.22 \\
\hline P6xP8 & $-1.38^{*}$ & $2.44^{* *}$ & 0.53 & -2.93 & -1.22 & -2.07 & 0.60 & 0.86 & 0.73 \\
\hline P7xP8 & 0.28 & $3.02^{* *}$ & $1.65^{* *}$ & -0.29 & $4.11^{*}$ & 1.91 & 1.25 & $3.91 * *$ & $2.58^{* *}$ \\
\hline LSD5\%(sij) & 1.05 & 0.94 & 0.70 & 4.05 & 3.50 & 2.64 & 2.00 & 1.94 & 1.37 \\
\hline LSD1\%(sij) & 1.40 & 1.25 & 0.91 & 5.38 & 4.64 & 3.46 & 2.65 & 2.57 & 1.80 \\
\hline LSD5\%(sij-sik) & 1.56 & 1.39 & 1.03 & 6.00 & 5.18 & 3.90 & 2.95 & 2.87 & 2.03 \\
\hline LSD1\%(sij-sik) & 2.07 & 1.85 & 1.35 & 7.96 & 6.87 & 5.12 & 3.92 & 3.80 & 2.66 \\
\hline LSD5\%(sij-ski) & 1.47 & 1.31 & 0.34 & 5.66 & 4.88 & 1.30 & 2.79 & 2.70 & 0.68 \\
\hline
\end{tabular}


E.H. El-Seidy, et al.,

\begin{tabular}{|c|c|c|c|c|c|c|c|c|c|}
\hline LSD1\%(sij-ski) & 1.95 & 1.74 & 0.45 & 7.50 & 6.47 & 1.71 & 3.69 & 3.58 & 0.89 \\
\hline
\end{tabular}

\begin{tabular}{|c|c|c|c|c|c|c|c|c|c|}
\hline \multirow[b]{2}{*}{ crosses } & \multicolumn{3}{|c|}{$\begin{array}{c}\text { Grain yield per } \\
\text { plant }\end{array}$} & \multicolumn{3}{|c|}{ Biological yield per plant } & \multicolumn{3}{|c|}{ Harvest index } \\
\hline & NS & $\mathbf{S}$ & Comb. & NS & $\mathbf{S}$ & Comb. & NS & $\mathbf{S}$ & Comb. \\
\hline P1xP2 & $-6.81^{* *}$ & $-2.54^{*}$ & $-4.67^{* *}$ & $8.51^{* \star}$ & -3.38 & 2.56 & $-11.78^{* \star}$ & -3.20 & $-7.49^{* *}$ \\
\hline P1xP3 & $-6.98 * *$ & -1.70 & $-4.34^{* *}$ & $-10.23^{* *}$ & -1.97 & $-6.10 * \star$ & $-6.96^{* \star}$ & -3.89 & $-5.43^{* *}$ \\
\hline P1xP4 & $-2.97^{* *}$ & $3.38^{* *}$ & 0.21 & $-12.89 * *$ & $10.32^{* *}$ & -1.29 & -0.01 & 0.93 & 0.46 \\
\hline P1xP5 & -1.31 & $-2.23^{*}$ & $-1.77^{*}$ & $-25.86 * *$ & $-6.20^{* *}$ & $-16.03^{* *}$ & $14.42^{* *}$ & -1.51 & $6.45^{* *}$ \\
\hline P1xP6 & $6.55^{* *}$ & $1.98^{*}$ & $4.27^{\star *}$ & -1.65 & $15.37^{\star *}$ & $6.86^{\star *}$ & $8.93^{\star *}$ & -3.36 & 2.79* \\
\hline P1xP7 & 0.45 & $2.04^{*}$ & 1.25 & $12.41^{* *}$ & 4.39 & $8.40^{\star *}$ & $-4.19^{*}$ & 1.42 & -1.38 \\
\hline P1xP8 & $2.90 * *$ & -1.00 & 0.95 & $8.20 * *$ & -3.92 & 2.14 & -0.08 & 0.10 & 0.01 \\
\hline P2xP3 & 1.29 & $-3.47^{* *}$ & \begin{tabular}{|l|}
$*$ \\
\end{tabular} & $-7.17^{* *}$ & -3.33 & $-5.25^{\star *}$ & $3.71^{*}$ & $-6.61^{* *}$ & -1.45 \\
\hline P2xP4 & $5.17^{* *}$ & $-3.56^{* *}$ & \begin{tabular}{l|l}
$*$ & 0.81 \\
\end{tabular} & $-6.42^{*}$ & -3.68 & $-5.05^{\star *}$ & $7.02^{\star *}$ & $-4.91^{*}$ & 1.06 \\
\hline P2xP5 & $3.17^{* \star}$ & $3.44^{\star *}$ & $3.31^{\star *}$ & $31.62^{\star *}$ & 4.42 & $18.02^{* *}$ & $-6.63^{\star \star}$ & $4.17^{*}$ & -1.23 \\
\hline P2xP6 & -1.77 & $3.47^{* *}$ & 0.85 & $16.37^{* \star}$ & -2.06 & $7.15^{\star \star}$ & $-6.56^{\star *}$ & $8.64^{* *}$ & 1.04 \\
\hline P2xP7 & $2.93^{* *}$ & $4.41^{\star *}$ & $3.67^{\star *}$ & 2.10 & $10.28^{\star \star}$ & $6.19 * *$ & 2.35 & 3.54 & $2.94^{\star}$ \\
\hline P2xP8 & -0.52 & 0.85 & 0.16 & $5.58^{*}$ & 3.14 & $4.36^{* \star}$ & -2.60 & -0.08 & -1.34 \\
\hline P3xP4 & -1.03 & $4.70^{* *}$ & $1.83^{* *}$ & $-17.40 * *$ & $35^{* *}$ & L.03 & $4.91^{* *}$ & 1.52 & 1.69 \\
\hline P3xP5 & $4.30 * *$ & $2.67^{* *}$ & $3.48^{\star *}$ & $6.44^{*}$ & $12.28^{* *}$ & $9.36^{* *}$ & 2.05 & -4.03 & -0.99 \\
\hline P3xP6 & $3.79 * *$ & 1.61 & $2.70^{\star *}$ & $8.18^{\star *}$ & $5.71^{*}$ & $6.94^{\star *}$ & 1.14 & -2.01 & -0.44 \\
\hline P3xP7 & -0.38 & 1.29 & 0.46 & 4.27 & -1.83 & 1.22 & -1.33 & 1.94 & 0.31 \\
\hline P3xP8 & 0.04 & $-4.33^{* *}$ & $\star-2.15^{* *}$ & $-9.09 * *$ & $6.43^{* *}$ & -1.33 & 3.58* & $-13.62^{\star *}$ & $-5.02^{* *}$ \\
\hline P4xP5 & $8.01^{* *}$ & -1.52 & $3.25^{* *}$ & $11.12^{* *}$ & 2.98 & $7.05^{* *}$ & 3.01 & $-4.54^{*}$ & -0.77 \\
\hline P4xP6 & 0.87 & $-3.10^{* \star}$ & \begin{tabular}{|l|} 
\\
\end{tabular} & $-11.97^{\star \star}$ & $-11.44^{\star *}$ & $-11.71^{\star *}$ & $4.78^{\star \star}$ & 0.63 & $2.71^{*}$ \\
\hline P4xP7 & -1.80 & $-2.22^{*}$ & $-2.01^{* *}$ & $-7.39 * *$ & $-12.74^{\star *}$ & $-10.07^{\star *}$ & 0.23 & 3.03 & 1.63 \\
\hline P4xP8 & $9.32^{\star \star}$ & $-3.59 * \star$ & * $2.86^{\star *}$ & $15.20^{\star *}$ & $-16.14^{\star \star}$ & -0.47 & $3.59^{*}$ & 3.67 & $3.63^{* *}$ \\
\hline P5xP6 & $-4.10 * *$ & $-3.95^{\star \star}$ & * $-4.03^{* *}$ & $6.08^{*}$ & $-5.28^{*}$ & 0.40 & $-7.46^{* *}$ & $-5.72^{* *}$ & $-6.59^{* *}$ \\
\hline P5xP7 & 1.57 & -0.20 & 0.68 & -1.66 & 1.35 & -0.16 & 1.94 & -1.46 & 0.24 \\
\hline P5xP8 & $3.35^{\star *}$ & $3.16^{* *}$ & $3.25^{\star *}$ & 0.84 & -2.17 & -0.67 & 2.92 & $8.95^{\star *}$ & $5.93^{* *}$ \\
\hline P6xP7 & $9.46 * *$ & $-2.22^{*}$ & $3.62^{* *}$ & -2.71 & $-14.25^{\star *}$ & $-8.48^{* *}$ & $11.90 * *$ & 4.02 & $7.96 * *$ \\
\hline P6xP8 & $-2.23^{*}$ & $2.89 * *$ & 0.33 & -0.02 & $8.96^{\star *}$ & $4.47^{\star *}$ & -2.86 & 0.63 & -1.11 \\
\hline P7xP8 & 0.37 & $5.05^{* *}$ & $2.71^{* *}$ & $17.86^{* *}$ & $8.41^{* \star}$ & $13.14^{\star *}$ & $-5.26^{* \star}$ & 4.58* & -0.34 \\
\hline LSD5\%(sij) & 1.96 & 1.92 & 1.35 & 5.01 & 4.43 & 3.29 & 3.33 & 4.10 & 2.60 \\
\hline LSD1\%(sij) & 2.60 & 2.55 & 1.77 & 6.64 & 5.88 & 4.32 & 4.41 & 5.43 & 3.41 \\
\hline LSD5\%(sij-sik) & 2.90 & 2.84 & 2.00 & 7.41 & 6.55 & 4.87 & 4.92 & 6.06 & 3.85 \\
\hline LSD1\%(sij-sik) & 3.84 & 3.77 & 2.62 & 9.83 & 8.70 & 6.39 & 6.53 & 8.04 & 5.04 \\
\hline
\end{tabular}


Combining ability and genetic component analyses for wheat under

\begin{tabular}{|l|l|l|l|l|l|l|l|l|l|}
\hline LSD5\%(sij-ski) & 2.73 & 2.68 & 0.67 & 6.99 & 6.18 & 1.62 & 4.64 & 5.71 & 1.28 \\
\hline LSD1\%(sij-ski) & 3.62 & 3.55 & 0.87 & 9.27 & 8.20 & 2.13 & 6.16 & 7.58 & 1.68 \\
\hline
\end{tabular}

*and **indicate significant at 0.05 and 0.01 levels of probability, respectively. NS= non stress, $\mathrm{S}=$ stress, $\mathrm{Com}=$ combined

For number of spikes/ plant, nine, five and eight crosses expressed significant and positive sij effects in normal irrigation, drought stress and the combined analysis, respectively. However, the best sij effects were detected for the cross $P_{3} \times P_{6}(6.79)$ in normal irrigation treatment, and combined analysis (3.58). For number of grains per spike, seven crosses in normal irrigation treatment, eleven crosses in drought stress condition and seven in the combined analysis expressed significant and positive sij effects. Moreover, the cross $P_{5} \times P_{7}$ gave the most desirable sij effects for this trait in normal irrigation (11.61), the cross P3 $X$ P5 in drought stress (15.00) and the cross P2 X P8 combined data (8.00). Regarding 1000-kernel weight, seven, seven and eight cross combinations expressed significant and positive ŝij effects in normal irrigation, drought stress and the combined data, respectively. The cross $P_{3} \times P_{8}$ gave the most desirable sij effects for 1000-kernel weight in normal irrigation, the cross P4XP8 in drought treatment, and the combined analysis being 5.25, 5.70 and 4.84, respectively. Eleven, eleven and eleven cross combinations exhibited significant and positive sij effects for grain yield I plant in non-stress, stress water environments, and the combined analysis, respectively. However, the best sij effects were detected for the crosses $\mathbf{P}_{6} \times \mathbf{P}_{7}, \mathbf{P}_{7} \times \mathbf{P}_{8}$ and $\mathbf{P}_{1} \times \mathbf{P}_{6}$ for the respective environments, respectively. Twelve, nine and eleven cross combinations exhibited significant and positive sij effects for biological yield/ plant in non-stress, stress environments, and the combined analysis. For harvest index, nine crosses in normal irrigation treatment, four cross in drought stress condition and seven in the combined analysis expressed significant and positive sijj effects. Moreover, the cross $P_{1} \times P_{5}$ gave the most desirable sij effects for this trait in normal irrigation (14.42), the cross P5 $\times$ P8 in drought stress (8.95) and the cross P6 $X$ P7 combined data (7.96).

\section{REFERENCES}

Abd El-Aty, M. S. M. and H. S. El-Borhamy (2007). Estimates of combining ability and susceptibility index in wheat diallel crosses under stress and normal irrigation treatments.Egypt. J. Plant Breed. 11(2): 651-667.

Akbar, M., J. Anwar, M. Hussain, M.H. Qureshi and S. Khan (2009). Linextester analysis in bread wheat (Triticum aestivum L.). J.Agric. Res. 47(1): 411.

Blum A (2006). Drought adaptation in cereal crops: A prologue. In: Ribaut $\mathrm{JM}$, editor. Drought Adaptation in Cereals. New York, NY, USA: Food Products Press, pp. 3-15

Dadbakhsh, A., A. Yazdansepas and M. Ahmadizadeh (2012). Influence of water deficit on yield and some quantitative traits in wheat genotypes .Curr. Res. J. of Biol. Sci., 4 (1): 75-81.

Dorostkar, S., A. Dadkhodaie and B. Heridari (2015). Evaluation of grain yield indices in hexaploid wheat genotypes in response to drought stress. Archives of Agronomy and soil Sci., 61 (3,4): 397-413.

EL-Hosary, A.A.A. and Gehan A. Nour EI Deen (2014). Genetic analysis in the $F_{1}$ and $F_{2}$ wheat generations of diallel crosses. Egypt. J. Plant Breed. 19 (2):355 -373 
EL-Hosary, A.A., M. E. M. EL-Badawy, A. K. Mustafa and M. H. EL-Shal (2012). Evaluation of diallel wheat crosses under drought tolerance. Egypt. J. Plant Breed. 16 (1): 19-40.

EL-Hosary, A.A., S.A. Sedhom, M.K. Khlifa and K. A. Bayoumi (2015). Heterosis, combining ability analysis for bread wheat under stress and normal irrigation treatments.The 9th Plant Breeding international Conference September 2015. Egypt. J. Plant Breed. 19 (5):87- 101.

Estehghari, M. R. and E. Farshadfar (2014). Evaluation of phenotypic variability, genetic parameters , heritability and genetic gain in bread wheat genotypes under rainfed conditions . Int. J. of Biosci., 4 (12): 193-201.

Farshadfar, E. (2012). Application of integrated selection index and rank sum for screening drought tolerant genotypes in bread wheat . Int. J. Agri. Crop Sci., 4 (6): 325-332.

Farshadfar, E., A. Ghaderi and A. Yaghotipoor (2014). Diallel Analysis of physiologic indicators of drought tolerance in bread wheat (Triticum aestivum L.). Agric. Communications. 2(1): 1-7.

Farshadfar, E., F. Rafiee and A. Yghotipoor (2012-a). Comparison of the efficiency among half diallel methods in the genetic analysis of bread wheat (Triticum aestivum L.) under drought stress condition. Ann. of Biological Res., 3 (3):1607-1622.

Farshadfar, E., F. Rafiee and $H$. Hasheminasab (2013). Evaluation of genetic parameters of agronomic and morpho-physiological indicators of drought tolerance in bread wheat (Triticum aestivum L.) usingdiallel mating design. Australian J. of Crop Sci., 7(2):268-275 .

Farshadfar, E., M. Allahgholipour, L. Zarei and M. Kiani (2011). Genetic analysis of field and physiological indicators of drought tolerance in bread wheat (Triticum aestivum L.)using diallel mating design. Afr. J. Biotechnol. 10(61):13071-13081.

Gomaa, M. A., M. N. M. El-Banna, A. M. Gadalla, E.E. Kandil and A.R.H. Ibrahim (2014). Heterosis, combining ability and drought susceptibility index in some crosses of bread wheat (Triticum aestivum L.) under water stress conditions . Middle- East j. Agric. Res., 3(2): 338-345.

Griffing, B. (1956). Concept of general and specific combining ability in relation to diallel crossing system. Aust J BiolSci 9: 463-493.

Hassan, W. A. A. (2007). Improving wheat production under saline and drought conditions by using diallel crossing system. Ph. D. Thesis. Fac. of Agric., Benha Univ., Egypt.

Monneveux, P. and J.M. Ribaut (2006). Secondary traits for drought tolerance improvement in cereals. In: Ribaut JM, editor. Drought Adaptation in Cereals. New York, NY, USA: Food Products Press, pp. 97-143.

Salgotra, R. K., B. B. Gupta and S. Praveen (2009). Combining ability studies for yield and yield components in Basmati rice. An International Journal on Rice. 46(1):12-16.

Sairam, R.K., P.S. Deshmukh, D.S. Shukla and S. Ram (1990). Metabolic activity and grain yield under moisture stress in wheat genotypes. Indian J Plant Physiol 33: 226-231 


\section{القدرة على التألف وتحليل مكونات التباين فى القمح تحث الإجهاد المائى والرى الطبيعى}

السيد حامد الصعيدى(') ، أمجد عبد الغفار الجمال(') ، رضا عحمد قنبر(")، هند مراجع عبدالله(')

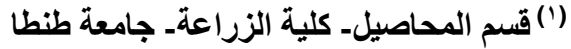

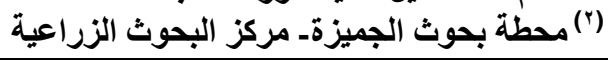

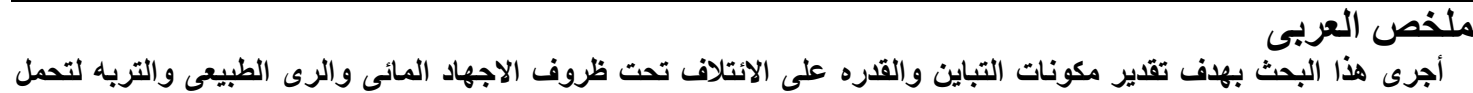

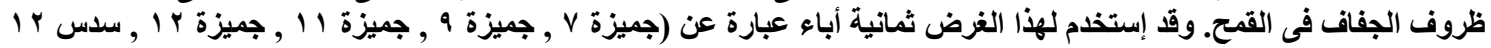

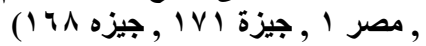

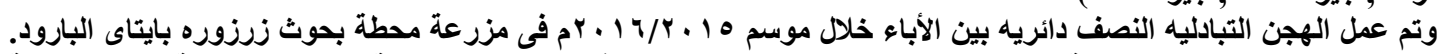

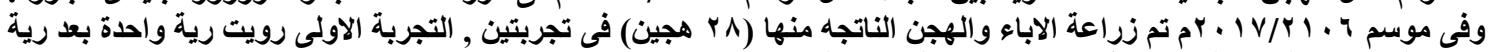

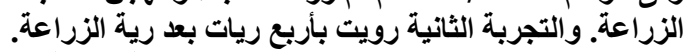
وكلا التجربتين مصممتان فى تصميم قطاعات كاملة النسبة ذو ثلاث مكررات وتم اخذ القياسات التاليه:

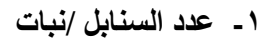

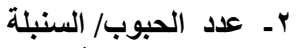

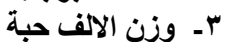

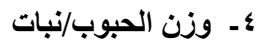

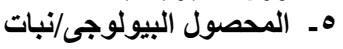

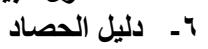

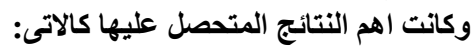
ا- كان التباين الراجع بين ظروف الرىى المختلفة معنوى جدا لكل الصفات المدروسه مما يدل على وجود إختلافات بين ظروف الإجهاد

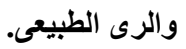
r- كما كان التباينى الراجع للاباء والهجن والتفاعل معنوى فى معظم الصفات المدروسه وايضا كان التباين الراجع للقدرة العامة

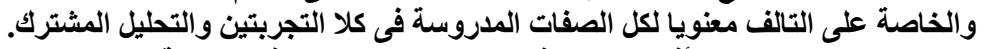

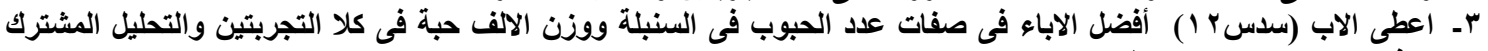

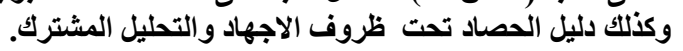

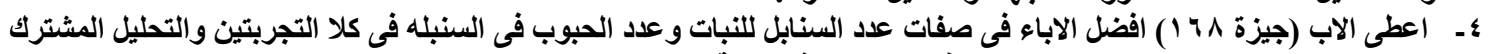

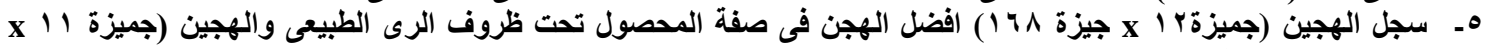

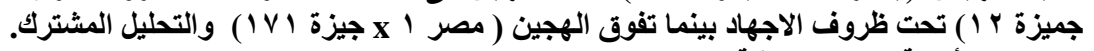
4- كما سجلت أربعة تراكيب وراثية وهى :

(P1*P6 , P2*P5 , P3*P5 , P8*P5)

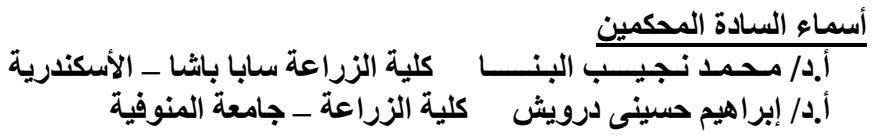

